\title{
Mathematical Modelling on the Effects of Acid Rain on a Fresh-Water Ecosystem
}

\author{
Mareta W. Ardyani
}

\begin{abstract}
The pH of a lake can be altered through the effects of acid rain (caused by pollution), and also can be seen to fluctuate over a period of time. This can have serious consequences on the ecosystem of the lakes and the biological species supported by the ecosystem are unable to survive if the pH level falls too low. This situation is of particular concern in lakes around the world because of pollution, and it is particularly serious in the Adirondack region in north eastern part of New York state. Most fresh-water ecosystems involve hundreds of interacting species, and modelling such a system is a rather difficult task. The objective of this project is to develop a fundamental understanding of the role of $\mathrm{pH}$ in a fresh-water ecosystem. A reasonably tractable prototype system could involve three species consisting of algae (chlorella), a herbivore (daphnia), and a predator (pumpkinseed sunfish). In this report we present the dynamics of the three species using logistic growth predator-prey model and perform stability analysis on the system with a quasi-steady state assumption. We model these interactions with a simple hydrogen ions model and perform simulation onto the coupled model with varied levels of the $\mathrm{pH}$ value.
\end{abstract}

Index Terms-acid rain, interacting species, predator-prey model, quasi steady state, hydrogen ions model

\section{INTRODUCTION}

Acid rain is a universal concern as it is a growing environmental problem. Naturally, rainwater should have a $\mathrm{pH}$ value that is at least 5.5, but in the north-eastern region of the United States, for example, the $\mathrm{pH}$ value of rain-water can drop to a level that is as low as 3.3. The increased acidity of the rain kills plants and animals in lakes and streams, causes increased weathering and decomposition of limestone in statues and on buildings, and affects the production of fruit and vegetable crops negatively.

Our focus is on the effects of acid rain on plants and animals in lakes and streams in the Adirondack region, which is located in north eastern New York state, USA. The region covers about $16,000 \mathrm{~km}^{2}$ (approximately $3 / 4$ area of Wales). It contains New York's highest peaks and it is heavily forested with approximately 1,300 lakes and ponds. Many of the lakes in the region are strongly affected by acid rain.[1]

One of the contributions to this is the utility plant pollution from the highly industrial Midwest states of Ohio, Illinois, Indiana and Pennsylvania. The gases emitted from the plants are carried north-east by wind patterns, as the wind rises over the Adirondack mountains, the moisture they contain cools and condenses into clouds. The moisture is saturated with

Mareta W. Ardyani, Department of Cryptography, Politeknik Siber dan Sandi Negara, Bogor, Indonesia. heavy amount of nitric and sulfuric acids which precipitate onto the Adirondack mountains, damaging the vegetation. Precipitation can be more than 200 times more acidic than natural rain. Now, more than 500 lakes and ponds (out of 2,800 ) in the Adirondack Park are already too acidic to support the plants and aquatic wildlife that once existed in them. Studies suggest that a $\mathrm{pH}$ level equal to about 6 is a critical biological threshold. A lake ecosystem can support many biological species when the $\mathrm{pH}$ level is greater than 6 . When the $\mathrm{pH}$ is below 6 , the number of lake species decreases and most species cannot exist if the $\mathrm{pH}$ falls below 4.5 as seen in Fig. 1.

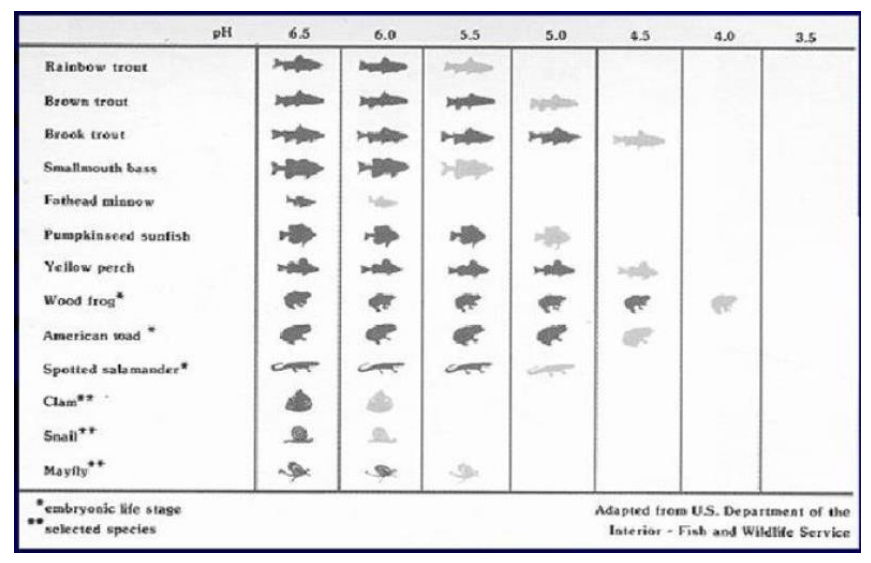

Fig. 1 Chart showing the vulnerability of fish in different $\mathrm{pH}$ level

Source: US Department of Interior - Fish and Wildlife Service

Our objective is to develop a fundamental understanding of the role of $\mathrm{pH}$ in a fresh-water ecosystem by creating a basic food web model which consists of a predator, a herbivore, and its food source (algae). The rates of growth, death and interaction depend, presumably, on the $\mathrm{pH}$ level of their environment. The hydrogen ions model will consist of the incoming and outgoing hydrogen ions and the lake buffering capacity. Our plans for this project were to develop and analyze a basic food-web (predator-prey) model and perform simulations for fixed $\mathrm{pH}$ and to develop and analyze a basic lake-pH model and perform simulations for different flow conditions. Next, we planned to develop and analyze a coupled food-web/lake-pH model and perform simulations for lake death or recovery. We also hope to determine short-time and long-time behaviors and calibrate models with available data, as best as possible.

\section{THE MODEL}

\section{A. The Hydrogen Ions Model}

The $\mathrm{pH}$ level of water in a lake is an important measure of 
the ability of the fresh-water ecosystem to support life. We can calculate $\mathrm{pH}$ by:

$$
p H=\log 10\left[H^{+}\right]
$$

where $\left[\mathrm{H}^{+}\right]$is the molar concentration of hydrogen ions (moles/liter). The hydrogen ion concentration in pure water at room temperature is about $10^{-7}$ moles/liter, thus natural $\mathrm{pH}$ is 7 .

Our first model was a linear model of hydrogen ions as given below:

$$
\frac{d H}{d t}=r_{\text {in }} H_{\text {in }}-r_{\text {out }} H-r_{\text {buf }}\left[H-H^{*}\right]
$$

with the following parameters:

$H$ - moles of hydrogen ions in the lake;

$H_{\text {in }}$ - moles of hydrogen ions in the incoming water;

$r_{i n}$ - incoming rate of moles of hydrogen ions;

$r_{\text {out }}$ - outgoing rate of moles of hydrogen ions;

$r_{\text {buf }}$ - buffering rate of the lake;

$H^{*}$ - maximum buffer limit

The maximum buffer limit is the highest level of hydrogen ions at which the lake can buffer. If the number of hydrogen ions exceeds $H^{*}$ then the lake can no longer buffer. Additionally, $r_{i n}$ represents the rate of incoming hydrogen ions from wet precipitation, surface runoff, ground water and inflow of water bodies into the lake and $r_{\text {out }}$ represents the rate of hydrogen ions leaving the lake through drainage and algae usage of $\mathrm{CO}_{2}$ during photosynthesis. We have chosen our buffering term to be the total number of hydrogen ions minus the maximum amount of hydrogen ions at which the lake can buffer. This term shows us a linear increase of the hydrogen ions.

We decided to change the way we modelled the buffering capacity of the lake. We know that many of the lakes in Adirondacks have very low buffering capacity [3] and this is due to the large increase in hydrogen ions coming into the lake which decreases the lake's buffering capacity rapidly. Thus, it is unlikely to be a linear relationship and we also know that the buffering capacity has a limiting value. To model this limiting behavior we considered using the form for buffering as below [12], and hence the dynamics of $H(t)$ is then governed by:

$$
\frac{d H}{d t}=r_{\text {in }} H_{\text {in }}-r_{\text {out }} H-B\left[\frac{H^{2}}{H^{*^{2}}+H^{2}}\right]
$$

In this equation, all parameters are the same as those defined above with $B$ is the buffering capacity of the lake.

\section{B. Food-Web Model}

We seek to model the food web model based on the interactions between the three species (fish, Daphnia and algae).

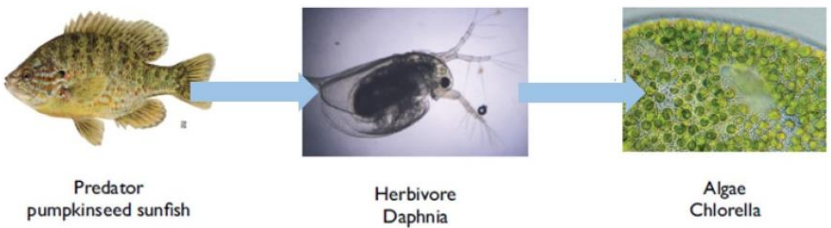

Fig. 2 Species interactions

Source: Oregon Department of Fish and Wildlife

The interactions between these three species can be seen from Fig. 2. The Pumpkinseed fish feed on Daphnia and the Daphnia then feed on the Chlorella (algae). The pumpkinseed fish reproduce rapidly and spawning occurs from May into August. The growth is fairly slow and is usually found in water less than 2.6 meter deep. Adult fish are able to survive low $\mathrm{pH}$ but the young ones will be affected by the decrease of $\mathrm{pH}$ level.

Daphnia are small water born crustaceans which have lifespan of about 50 days. Larger Daphnia avoid predation by fish by moving away and hiding into the lower portion of the lake. Chlorella (algae) grows rapidly where the population doubles in approximately 14 hours. Chlorella are able to remove $\mathrm{CO}_{2}$ through the process of photosynthesis hence increase the $\mathrm{pH}$ level of the lake.

There are several assumptions that we made before developing our predator-prey model. First assumption is that the predator is dependent on the prey population, which means that the prey is the only source of food and the predators do not feed on other species. In addition, the prey has no threat in their life other than being hunted by predators. The rate at which predator encounter prey is directly proportional to both populations. We also take a fixed proportion of encounters between prey and predator leading to death of prey.

We have our food-web model with logistic growth as such in the following equations:

$$
\begin{gathered}
\frac{d F}{d t}=-a F+b D F \\
\frac{d D}{d t}=c D-\gamma D^{2}-d D F+f D A \\
\frac{d A}{d t}=g A-\sigma A^{2}-h D A
\end{gathered}
$$

The initial conditions are $F(0)=F_{0} \geq 0, A(0)=A_{0} \geq 0$, and $D(0)=D_{0} \geq 0$. The parameters $a, b, c, d, f, g, h, \gamma$, and $\sigma$ are all positives and as such:

Pumpkinseed fish - denoted by $F=F(t)$ is the number of fish population;

Daphnia - denoted by $D=D(t)$ is the number of Daphnia population;

Algae - denoted by $A=A(t)$ is the number of algae populations; 
$a$ - linear death of Pumpkinseed fish;

$b$ - rate of predation of Pumpkinseed fish;

$c$ - linear birth rate of Daphnia;

$d$ - rate at which Daphnia are killed due to encounters with fish;

$f$ - rate of predation of Daphnia;

$g$ - linear birth rate of algae;

$h$ - rate at which algae are killed due to encounters with Daphnia;

$\gamma$ - logistic term of Daphnia;

$\sigma-$ logistic term of algae.

Next, we perform analysis on each of the hydrogen ions model and the food-web model.

\section{ANALYSIS OF THE MODELS}

\section{A. Analysis of the Hydrogen Ions Model}

To analyze the model, it is essential to express it in nondimensional terms. This will enable us to reduce the number of relevant parameters to dimensionless groupings which determine the dynamics. Therefore, we introduce nondimensional quantities by writing:

$$
\tau=\frac{B t}{H^{*}}, \quad \widehat{H}=\frac{H}{H^{*}}, \quad \alpha=\frac{H^{*} r_{\text {out }}}{B}, \quad \beta=\frac{r_{\text {in }} H_{\text {in }}}{B}
$$

which upon substitution into the original model becomes:

$$
\frac{d \widehat{H}}{d \tau}=\beta-\alpha \widehat{H}-\left(\frac{\widehat{H}^{2}}{1+\widehat{H}^{2}}\right)
$$

The steady state are solutions of:

$$
\beta-\alpha \widehat{H}-\left(\frac{\widehat{H}^{2}}{1+\widehat{H}^{2}}\right)=0
$$

Although we know the analytical solutions of a cubic equation [13], they are often awkward to use because of their algebraic complexity; this is one of these cases. We explored the steady state solution graphically and analytically for our model and came to the conclusion that we needed a simpler model for the hydrogen ions, especially since we wanted to introduce this model into the food-web model afterwards.

We next considered a Michaelis-Menten kinetics form [14] for the buffering capacity. This gives us:

$$
\frac{d H}{d t}=r_{\text {in }} H_{\text {in }}-r_{\text {out }} H-B\left[\frac{H}{H^{*}+H}\right]
$$

We used the same nondimensional quantities as before and our new equation becomes:

$$
\frac{d \widehat{H}}{d \tau}=\beta-\alpha \widehat{H}-\left(\frac{\widehat{H}}{1+\widehat{H}}\right)
$$

The steady states are the solutions of:

$$
\beta-\alpha \widehat{H}-\left(\frac{\widehat{H}}{1+\widehat{H}}\right)=0
$$

which are:

$$
\widehat{H}=\frac{\beta-\alpha-1}{2 \alpha} \pm \sqrt{\frac{(\beta-\alpha-1)^{2}}{4 \alpha^{2}}+\frac{\beta}{\alpha}}
$$

Plotting the quadratic function at various values of $\alpha$ and $\beta$, we found that both solutions were stable steady states since at both roots the derivative is negative. We chose the positive square root solution to use in the coupled model.

\section{B. Analysis of the Food-Web Model}

We would like to analyze the food-web model that we have developed in the system governing the three equations in the model. We run simulation of this system in Matlab using ode45 and observe the solutions. We obtained the graph in Fig. 3.

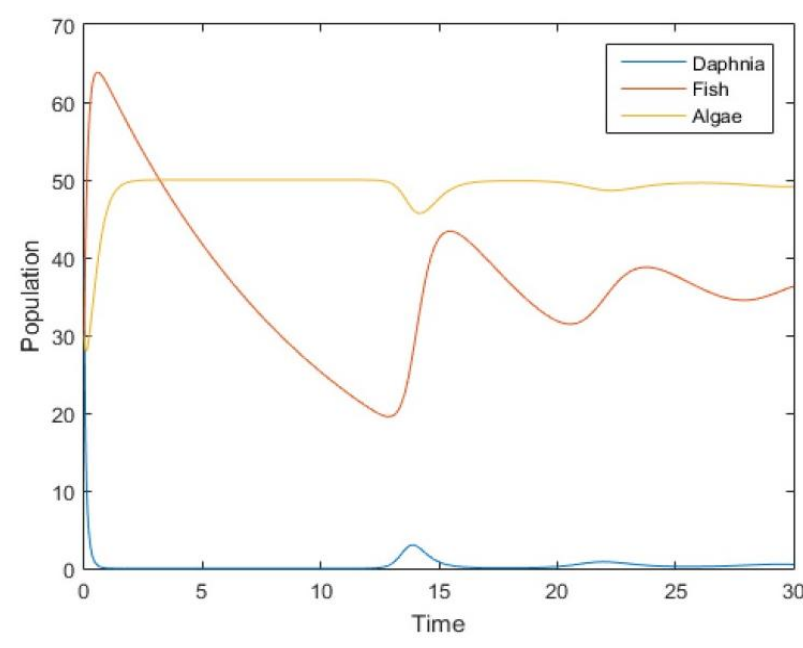

Fig. 3. Matlab simulations of the food-web model with $a=0.1, b=0.25, c=5, \gamma=0.6, d=0.2, f=0.05$, $g=3, \sigma=0.06$, and $h=0.1$

One can observe from Fig. 3 that the population of the fish increases drastically at the beginning of time because they are receiving predation benefits by feeding onto the Daphnia and hence their population grows. However, this reduces the population of Daphnia at the same time. Observe that the population of algae increases as the population of Daphnia decreases but it only increases up to a certain point due to the logistic term of our system.

We proceed with examining the solution to our system by making a quasi-steady-state assumption and assume that the algae grow rapidly and does not change at the timescale that we are investigating. Now this leads to the rate of change of algae population equals to zero giving us an expression with A.

$$
\frac{d A}{d t}=g A-\sigma A^{2}-h D A=0
$$




$$
A=\frac{g-h D}{\sigma}
$$

The expression can be substituted into our Pumpkinseed fish and Daphnia equations, resulting in:

$$
\frac{d F}{d t}=-a F+b D F
$$

$$
\frac{d D}{d t}=c D-\gamma D^{2}-d D F+f D\left(\frac{g-h D}{\sigma}\right)
$$

Simplifying the Daphnia equation gives us:

$$
\frac{d D}{d t}=\left(c+\frac{f g}{\sigma}\right) D-\left(\gamma+\frac{f h}{\sigma}\right) D^{2}-d D F
$$

Letting $\alpha=\left(c+\frac{f g}{\sigma}\right)$ and $\xi=\left(\gamma+\frac{f h}{\sigma}\right)$, we obtained a simplified system of Pumpkinseed fish and Daphnia as follows:

$$
\begin{gathered}
\frac{d F}{d t}=F(-a+b D) \\
\frac{d D}{d t}=D(\alpha-\xi D-d F)
\end{gathered}
$$

We then proceeded with finding the equilibrium points of the system above and obtained the three possible equilibrium points $E_{i}=(F, D)_{(i=1,2,3)}$ :

$$
\begin{gathered}
E_{1}=(0,0) \\
E_{2}=\left(0, \frac{\alpha}{\xi}\right) \\
E_{3}=\left(\frac{\alpha b-\xi a}{b d}, \frac{a}{b}\right)
\end{gathered}
$$

Note that the third equilibrium point only exists if $\alpha b-\xi a>0$.

If we make a small perturbation about the critical points, we can analyze the stability of these equilibrium points using the Jacobian method [14] by analyzing its eigenvalues. We obtain:

Table I. Summary of stability of the three equilibrium points

\begin{tabular}{|c|c|c|}
\hline Cases & Equlibrium Points & Stability \\
\hline \multirow{3}{*}{$b>a$} & $E_{1}=(0,0)$ & $\begin{array}{c}\text { Unstable (saddle } \\
\text { point) }\end{array}$ \\
\cline { 2 - 3 } & $E_{2}=\left(0, \frac{\alpha}{\xi}\right)$ & Unstable \\
\cline { 2 - 3 }$b<a$ & $E_{3}=\left(\frac{\alpha b-\xi a}{b d}, \frac{a}{b}\right)$ & Stable focus \\
\hline \multirow{3}{*}{$b$} & $E_{1}=(0,0)$ & Unstable \\
\cline { 2 - 3 } & $E_{2}=\left(0, \frac{\alpha}{\xi}\right)$ & Stable \\
\cline { 2 - 3 } & $E_{3}$ does not exist & - \\
\hline
\end{tabular}

We can observe that when the death rate of the fish is greater than the predation benefits, all solutions go toward second equilibrium point $\left(E_{2}=\left(0, \frac{\alpha}{\xi}\right)\right)$ where the fish population decreases to 0 as $t$ tends to $\infty$. On the other hand, when the predation benefits is greater than the death rate of fish, all solutions go toward third equilibrium point $\left(E_{3}=\right.$ $\left(\frac{\alpha b-\xi a}{b d}, \frac{a}{b}\right)$ ) where population are maintained, both Pumpkinseed fish and Daphnia survive. As t tends to $\infty, F$ tends to $\frac{\alpha b-\xi a}{b d}$ and $D$ tends to $\frac{a}{b}$.

The results obtained in Table I can be seen from demonstrations of the phase portrait of the system using Matlab in Fug. 4 and Fig. 5.

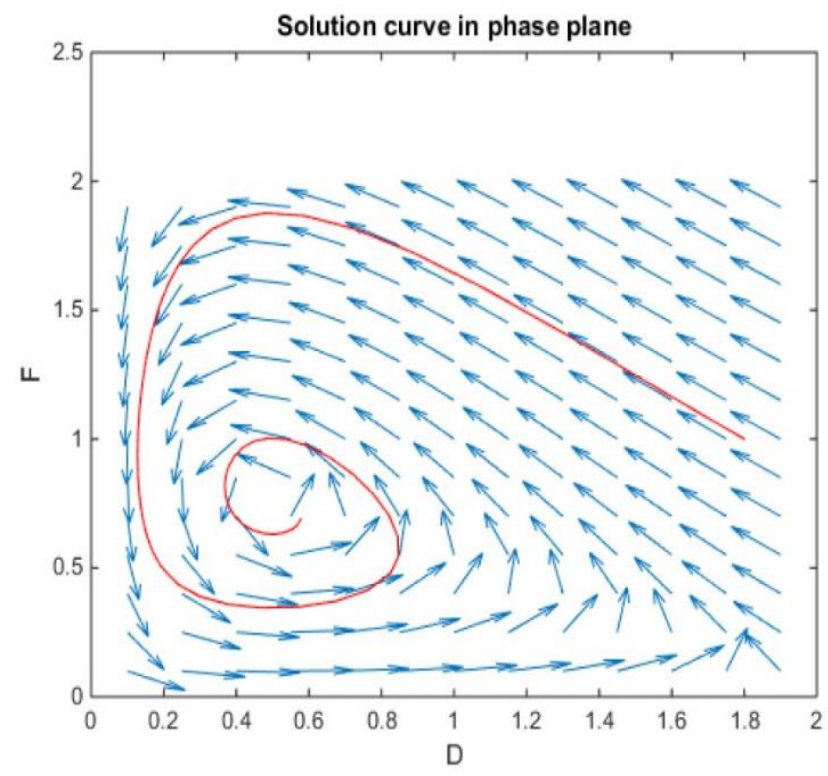

Fig. 4 . The case where $b>a$, which produces a stable focus phase portrait and all solutions go toward $E_{3}=\left(\frac{\alpha b-\xi a}{b d}, \frac{a}{b}\right)$

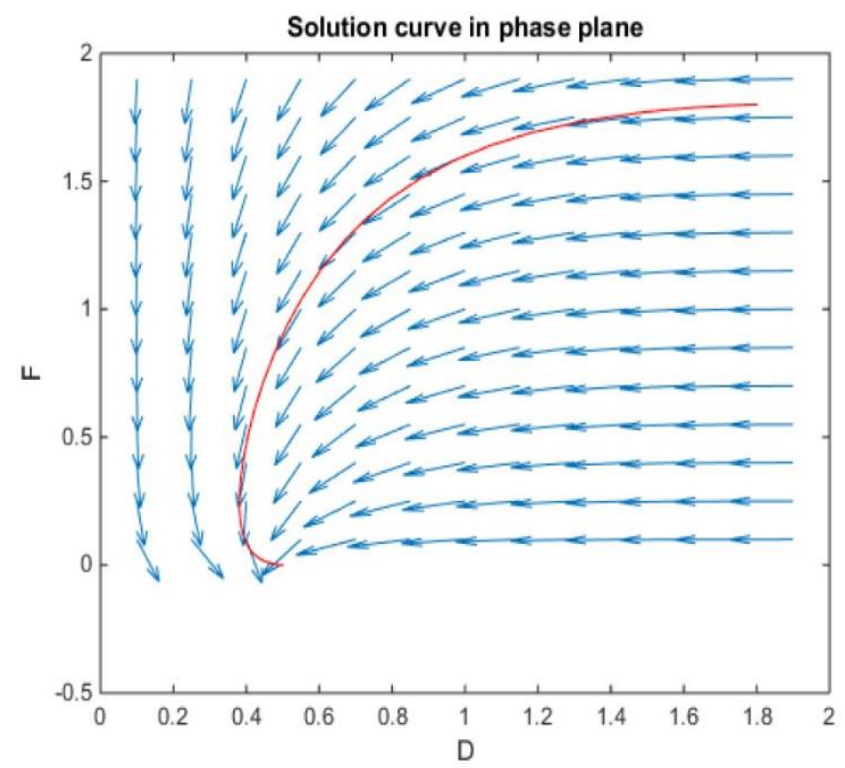

Fig. 5. The case where $b<a$, which produces a stable node phase portrait and all solutions go toward $E_{2}=\left(0, \frac{\alpha}{\xi}\right)$ 


\section{INCORPORATING THE FOOD-WEB AND HYDROGEN IONS MODELS}

In the previous section, we have established that the equation for algae is no longer included in our system based on the quasi-steady state assumption. We have solved the equation for algae and obtained the following expression:

$$
A=\frac{g-h D}{\sigma}
$$

Hence, this results in the simple system of only the Pumpkinseed fish and the Daphnia.

The $\mathrm{pH}$ level of a lake has a great influence on the viability of organisms living in it. Recall that, as the amount of hydrogen ions in a lake gets larger, the $\mathrm{pH}$ level of the lake will go down. The low $\mathrm{pH}$ level will bring harm to aquatic lives within the lake. Many literatures related to this topic have wide explanations on how low $\mathrm{pH}$ level can kill living organism faster [9]. We believe that low $\mathrm{pH}$ level can also harm other organisms within the lake (i.e., Daphnia). Moreover, we considered the possibility that low $\mathrm{pH}$ level might also poison the fish which then might result in decreasing reproduction rate and eventually might decrease the birth rate of the fish. However, since we have looked at many literatures which only focus on how $\mathrm{pH}$ level harm the viability of the fish, we decide to focus on the same thing. We have solved the hydrogen ions model and got the following expression for $H$ :

$$
H=H^{*} \widehat{H}
$$

where $H^{*}$ is the threshold where the lake starts to buffer, and $\widehat{H}$ is given by:

$$
\widehat{H}=\frac{\beta-\alpha-1}{2 \alpha} \pm \sqrt{\frac{(\beta-\alpha-1)^{2}}{4 \alpha^{2}}+\frac{\beta}{\alpha}}
$$

Since we have obtained $H$, we then incorporated the equation for hydrogen ions with the equation for fish by substituting the value of $H$ into the death rate of the fish. Hence, our system has now become:

$$
\begin{gathered}
\frac{d F}{d t}=-\hat{a} H F+b D F \\
\frac{d D}{d t}=D(\alpha-\xi D-d F)
\end{gathered}
$$

with $a$, the death rate of the fish, given by:

$$
a=\hat{a} H
$$

where $a$ is some constant.

As the amount of hydrogen ions increases, $\mathrm{pH}$ level decreases, and thus the death rate of the fish increases. Conversely, when the amount of the hydrogen ions decreases, $\mathrm{pH}$ level increases and thus the death rate of the fish will decrease.

As a result, when the amount of hydrogen ions increases, $E_{1}$ becomes unstable, $E_{2}$ becomes stable, and $E_{3}$ does not exist since we cannot have populations to be negative. This means that all solutions will go to $E_{2}$ where Daphnia survive but fish die out due to the increase of death rate caused by $\mathrm{pH}$ level. On the contrary, when the amount of hydrogen ions decreases, $E_{1}$ and $E_{2}$ become unstable, and $E_{3}$ becomes stable and exists. This means that all solutions will go to $E_{3}$, where both fish and Daphnia survive and the $\mathrm{pH}$ level does not affect the populations.

\section{A. Performing Simulations on the Coupled Model}

We then performed simulations on Matlab using ode 45 to capture the behavior of our models when we put them together. This simulation includes the hydrogen terms affecting the death rate of our fish population. When the hydrogen ions increase, the term $a=\hat{a} H$ also increases, hence $b<a$ (i.e., the death rate of the fish is greater than predation benefit), then the fish will eventually die out due to low $\mathrm{pH}$ level, and as a result, the number of Daphnia will grow considerably larger, even though at the beginning of the simulation the number of Daphnia decreases extremely due to high predation by the fish, as shown in Fig. 6 .

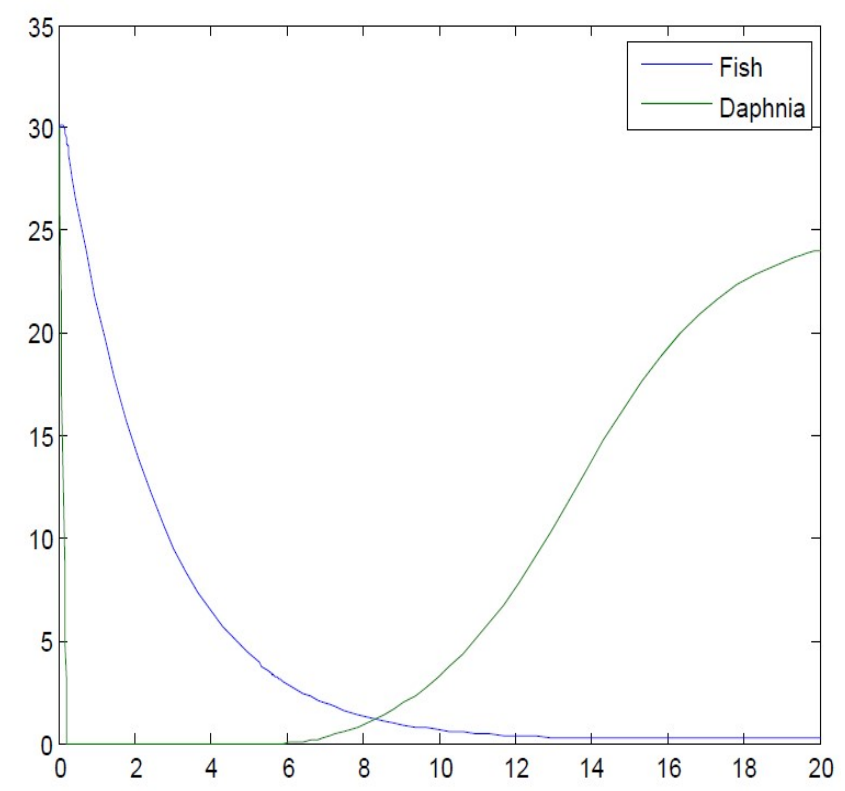

Fig. 6. Matlab simulation of the behavior of the coupled models when $b<a$ (i.e., the death rate of the fist is greater than the predation benefits)

Similarly, when the amount of hydrogen ions decreases, $a=\hat{a} H$ decreases and $b>a$ (i.e., the death rate of the fish is less than the predation benefit), it is predicted that, both populations will survive (at the timescale we are investigating). This can be shown in Fig. 7 below. 


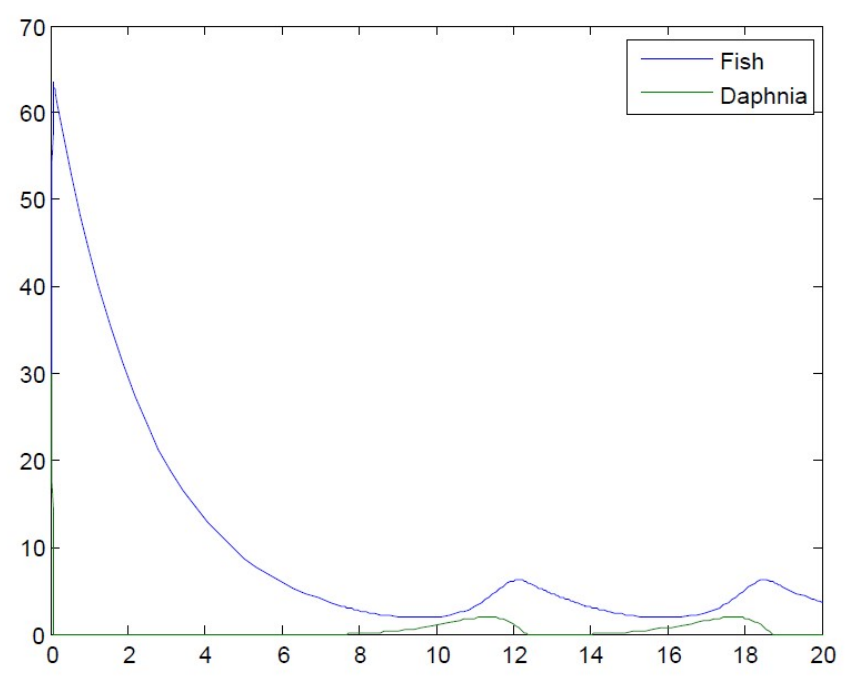

Fig. 7. Matlab simulation of the behavior of the coupled models when $b>a$ (i.e., the death rate of the fish is less than the predation benefits)

\section{DISCUSSION}

\section{A. Results}

We developed a food web model of the Pumpkinseed fish, Daphnia and algae populations and also developed a hydrogen ions model and coupled both models so we could analyze the effect of the $\mathrm{pH}$ level on the species in the lake. Simulations were performed on the model with varied $\mathrm{pH}$ levels and at the end of our analysis we have two possible situations:

- The survival of only the Daphnia population for a low $\mathrm{pH}$ level.

- Both the Daphnia and Fish population co-exist when there is an increase in the $\mathrm{pH}$ value.

\section{B. Problems Encountered}

Some of the problems we encountered during the project include:

- Finding the proper mathematical representation of the buffering behavior of the lake has proved to be tricky, as seen in equations (3), (7), (8) and (9). These results would have caused complications if we attempted to merge them with the food-web model, so in order to keep our model simple and easy to analyze, we chose to make use of a model which although works for only a limited $\mathrm{pH}$ value range, eliminates the complication.

- In the attempt to couple the food web and hydrogen ions model, we discovered that the introduction of the $\mathrm{pH}$ term to the entire population concealed the effect the $\mathrm{pH}$ level had on the individual population. Since we wanted to study how the $\mathrm{pH}$ level affects each population so we decided to introduce the $\mathrm{pH}$ effect to one population at a time.

- We attempted to analyze an age structured model. The fish population was split into young and mature fish.

Let $Y=$ young fish, $M=$ mature fish, $D=$ Dapnia, $A=$ Algae, where the total fish population $F(t)=M(t)+$ $Y(t)$.

$$
\begin{gathered}
\frac{Y}{t}=a M+b Y D-c Y-e Y \\
\frac{d M}{d t}=e Y+g M D-h M
\end{gathered}
$$

$$
\frac{d D}{d t}=i D\left(1-\frac{D}{K}\right)+n A D-j M D-l Y D
$$

$$
\frac{d A}{d t}=q A\left(1-\frac{A}{k}\right)-r D A
$$

$a$ - linear birth rate of young fish;

$b$ - rate of predation of young fish;

$c$ - death rate of young fish;

$e$ - rate at which young fish grow to maturity;

$g$ - rate of predation of mature fish;

$h$ - death rate of mature fish;

$i$ - logistic growth rate of Daphnia;

$n$ - rate of predation of Daphnia;

$j$ - death rate of Daphnia from encounter with mature fish;

$l$ - death rate of Daphnia from encounter with young fish;

$q$ - logistic growth rate of algae;

$r$-death rate of algae due to encounter with Daphnia.

Adopting similar conditions with our earlier solution in equations (14) to (18), we made a quasi-steady state assumption and reduced the system to three ordinary differential equations.

$$
\frac{Y}{t}=a M+b Y D-c Y-e Y
$$

$$
\frac{d M}{d t}=e Y+g M D-h M
$$

$$
\frac{d D}{d t}=u D-v D^{2}-j M D-l Y D
$$

Analyzing the systems at steady state we found an expression for $Y$ :

$$
\begin{gathered}
e Y+g M D-h M=0 \\
Y=\frac{h M-g M D}{e}
\end{gathered}
$$

and substituted the value to get a reduced system of equations:

$$
\begin{gathered}
x M+y M D-z M D^{2}=0 \\
u D-v D^{2}-z M D+\phi M D^{2}=0
\end{gathered}
$$

We solved the reduced system at steady state and found 
three equilibrium points $E_{i}=(D, M)_{(i=1,2,3)}$

$$
\begin{gathered}
E_{1}=(0,0) \\
E_{2}=\left(\frac{u}{v}, 0\right) \\
E_{3}=\left(\rho, \frac{u-v \rho}{z-\phi \rho}\right)
\end{gathered}
$$

$E_{1}$ resulted in an unstable state but this is from a trivial equilibrium point. $E_{2}$ had a stable point but with zero fish population and a constant Daphnia population this was not the result we were hoping for. $E_{3}$ had a complex algebraic expression that could not be defined as stable or unstable.

We decided that this was probably not the best method to use in analyzing the system. There are other methods which could produce better results that will help in the understanding and evaluation of the age structured model. Suggested methods include the use of difference equations and solving the system of differential equations simultaneously with the introduction of constant values to represent the rates in the equation.

\section{Future Work}

Our project so far has only considered the effect of $\mathrm{pH}$ on the death rate of the fish population, further study can be carried out on the effect of the $\mathrm{pH}$ on the Daphnia and algae population. Future study can take into account the $\mathrm{pH}$ variations that occur due to seasonal changes during the year. The $\mathrm{pH}$ level also changes during the day due to photosynthesis, which can also be analyzed. The long term effect of liming can also be studied to see how it aids the improvement of the ecosystem.

\section{REFERENCES}

[1] J. Jenkins, K. Roy, C. Driscoll, C. Buerkett. Acid Rain and the Adirondacks: A Research Summary, Ray Brook, 2005.

[2] C. T. Driscoll, G. B. Lawrence, A.J. Bugler, et.al. Acid Rain Revisited: Advances in Scientific Understanding Since the Passage of the 1970 and 1990 Clean Air Act Amendments., Science Links, 2001, Vol. 1, no.1.

[3] C.T. Driscoll, K.M. Driscoll, Karen M. Roy, M. J. Mitchell. Chemical Response of Lakes in the Adirondack Region of New York to Declines in Acidic Deposition, Environmental Science and Technology, 2003 vol.37(10), pp. 2036-2042.

[4] M. Manca, J. Vijverberg, L. Polishchuk, D. Voronov. Daphnia Body Size and Population Dynamics Underpredation by Invertebrate and Fish Predators in Lago Maggiore: An Approach Based on Contribution Analysis, Journal of Limnology, 2008, Vol.67(1), pp.15-21.

[5] L. Wu, D. Culver. Daphnia Population Dynamics in Western Lake Erie: Regulation by Food Limitation and Yellow Perch Predation, Journal of Lake Research, 1994, Vol.20(3), pp. 537-545.

[6] J. Grasman, E. van Nes and K. Kersting.Data-Directed Modelling of Daphnia Dynamics in a Long-term Micro-ecosystem Experiment, Ecological Modelling, 2008, Vol. 220(3), pp.343-350

[7] J. Geelen and R. Levven. Impact of Acidification on Phytoplankton and Zooplankton Communities, Cellular and Molecular Life Sciences, 1986, Vol.42(5), pp. 486-494

[8] E. McCauley, R. Nisbet, W. Murdoch. Large-Amplitude Cycles of Daphnia and its Algal Prey in Enriched Environments, Nature, 1999, Vol. 402(9), pp. 653-656.

[9] M. Rask. The Effect of Low pH on perch, Perca Fluviatilis L.II. The Effect of Acid Stress on Different Development Stages of Perch, Annales Zoologici Fennici, 1984, Vol. 21(1), pp.9-13.

[10] S. Gloss, C. Schofield, M. Marcus. Liming and fisheries management guidelines for acidified lakes in the Adirondack Region, National Ecology Research Center, Biological Report,1989, 80(40.27), xi + 59 pp.
[11] D. Knappe. Algae Detection and Removal Strategies for Drinking Water Treatment Plants, American Water Works Association, 2004.

[12] D. Ludwig, D. Jones, C. Holling. Qualitative Analysis of Insect Outbreak Systems: The Spruce Budworm and Forest, Journal of Animal Ecology, 1978, Vol. 47(1), pp. 315-332.

[13] L. Edelstein-Keshet. Mathematical Models in Biology, Society for Industrial and Applied Mathematics, 2005.

[14] J. Murray. Mathematical Biology, Springer Books, 2002. 